\title{
Psychometric Properties of a Portuguese Version of the Subjective Happiness Scale
}

\author{
Paola Spagnoli • António Caetano • Ana Silva
}

Accepted: 27 November 2010/Published online: 5 December 2010

(C) Springer Science+Business Media B.V. 2010

\begin{abstract}
The Subjective Happiness Scale (SHS) constitutes an instrument for assessing subjective happiness. This study aims to present the validation of the SHS in a Portuguese adult population. A large representative sample (1,017 participants), from five different age groups was considered. Configurational invariance of the unidimensional structure of the scale was tested through multi-group confirmatory factorial analysis, and reliability and criterion-validity were investigated. The results support the single factorial structure and reliability of the SHS. Furthermore, the SHS correlated positively with the Satisfaction with Life Scale, thus supporting its criterion validity. Therefore, the Portuguese version of the SHS is a valid instrument to use with an adult Portuguese Sample.
\end{abstract}

Keywords Subjective well-being - Satisfaction with Life Scale ·

Subjective Happiness Scale · Validity · Portugal

\section{Introduction}

The last three decades have witnessed increasing interest in research on happiness and subjective well-being from a variety of perspectives; psychology, sociology, gerontology and economy. Growing attention to this topic has encouraged the proliferation of several definitions and measures of it (e.g., Bradburn 1969; Diener et al. 1985; Dupuy 1978; Lyubomirsky and Lepper 1999).

One of the most widely used measures of happiness is Bradburn's (1969) Affect Balance Scale, which assesses the balance of positive and negative affects experienced during the previous 4 weeks. Thus, the Affect Balance Scale is a measure of the affective component of subjective well-being.

P. Spagnoli · A. Caetano - A. Silva

ISCTE-IUL, Lisbon University Institute, Lisbon, Portugal

P. Spagnoli $(\bowtie)$

CIS Centro de Investigação e Intervenção Social ISCTE-IUL Avenida

das Forças Armadas, Edifício ISCTE, 1649-026 Lisbon, Portugal

e-mail: paola.spagnoli@iscte.pt 
Another of the most used measures of subjective well-being, the Satisfaction With Life Scale (SWLS; Diener et al. 1985), assesses the concept of life satisfaction by measuring individuals' global judgement of their lives and, thus, the cognitive component of subjective well-being. According to Gouveia et al. (2009) this scale has been used in more that 4,000 studies and has been translated into several languages (e.g., Abdallah 1998; Arrindell et al. 1991; Blais et al. 1990; Gouveia et al. 2009; Neto 1993).

Other measures of subjective well-being include single-item scales, such as Bradburn's (1969) Global Happiness Item or scales that tap into various aspects of psychological functioning in addition to well-being (e.g., Dupuy 1978). The most recent Subjective Happiness Scale (Lyubomirsky and Lepper 1999) assesses the overall "subjective happiness", covering the gap in the literature regarding the need for a measure focused on whether one is a happy or an unhappy person. Lyubomirsky and Lepper (1999) posited “.... such a measure would reflect a broader and more molar category of well-being and tap into more global psychological phenomena (Diener 1994). ...most individuals are capable of reporting on the extend to which they are a happy person (or unhappy one), and this judgement is likely not equivalent to a simple sum of their recent levels of affect and their satisfaction with life (p. 140)".

Although the Subjective Happiness Scale is an integral instrument for assessing individual well-being, the research currently available reports the validation of the scale in just a few populations: the North American and the Russian populations (Lyubomirski and Lepper 1999), Japanese (Shimai et al. 2004), Chinese and Malays (Swami 2008), Austrian and Tagalog (Swami et al. 2009). Research, therefore, is needed to validate the SHS in other countries. In particular, a validation of the scale in a Latin language country is still lacking. According to Swami et al. (2009), the translation and the validation of the SHS for use in diverse samples will aid cross-cultural research on subjective happiness. Research in this field pointed out that although happiness is valued in different cultures (e.g., Diener and Diener 1995; Ryan et al. 1999) there might be cross-cultural differences in the meaning of happiness and variables associated with happiness (Swami et al. 2009). Thus, the aim of the present paper is to present the psychometric properties of the Portuguese version of the Subjective Happiness Scale by assessing its construct validity across five diverse groups of Portuguese working adults balanced for age and gender. Evidence of the relationship between the construct of Life Satisfaction and Happiness in the Portuguese culture is also provided. This evidence provides information regarding the criterion-validity of the SHS.

\section{Method}

\subsection{Sample}

A total of 1,017 participants from five age groups took part in this study. The 1,017 participants represent the Portuguese working adult population balanced for age and gender and correspond to five age groups: from 25 to 29 years old; from 30 to 34 years old; from 35 to 39 years old; from 40 to 44 years old; from 45 to 50 years old. A stratified sampling method using proportionate allocation strategy was used to collect the data. Table 1 provides information for each group.

\subsection{Instruments}

The questionnaire including the Satisfaction With Life Scale (SWLS), the Subjective Happiness Scale (SHS) and demographics questions, was administered by telephone to all 
Table 1 Groups and overall demographics and Cronbach's alphas for SHS

\begin{tabular}{lrlllll}
\hline Groups & $N$ & Age range & Female (\%) & Alpha & M & SD \\
\hline 1 & 206 & $25-29$ & 52 & .80 & 4.84 & 1.08 \\
2 & 201 & $30-34$ & 47 & .73 & 4.73 & 1.01 \\
3 & 209 & $35-39$ & 48 & .77 & 4.64 & 1.08 \\
4 & 200 & $40-44$ & 59 & .79 & 4.79 & 1.07 \\
5 & 201 & $45-50$ & 59 & .76 & 4.85 & 1.12 \\
Overall & 1,017 & $25-50$ & 53 & .77 & 4.77 & 1.08 \\
\hline
\end{tabular}

the participants. The SWLS was included in the study in order to test the criterion validity of the SHS.

\subsubsection{SWLS}

The five items of the SWLS (Diener et al. 1985) were answered according to a 7-point scale, ranging from $1=$ strongly disagree to $7=$ strongly agree.

\subsubsection{SHS}

The items of the SHS are: (1) "In general, I consider myself..." Respondents used a 7point scale, ranging from $1=$ not a very happy person to $7=$ a very happy person, (2) "Compared to most of my peers, I consider myself..." Respondents chose from $1=$ less happy to $7=$ more happy, (3) "Some people are generally very happy. They enjoy life regardless of what is going on, getting the most out of everything. To what extent does this characterization describes you?" Responses were made on a 7-point scale, ranging from $1=$ not at all to $7=$ a great deal, (4) "Some people are generally not very happy. Although they are not depressed they never seem as happy as they might be. To what extent does this characterization describes you?" Responses were made on a 7-point scale, ranging from $1=$ not at all to $7=$ a great deal. This item is reversed. The Portuguese version of SHS is available from the authors.

\subsection{Procedure}

To ensure equivalence of meaning of the items between the Portuguese and English versions of the SHS, a rigorous translation process was used that included forward and backward translation and pilot testing. The translation process began with the translation of the English version into Portuguese by a translator bilingual in English and Portuguese. After completing the forward translation another bilingual translator (a native English speaker) independently translated the SHS back into English. The translators then compared the back translation to assess the item-by-item consistency. Before the SHS application, a pilot test was conducted in order to gather feedback on the readability and content validity of the translated instrument. This instrument was applied to 15 individuals and no significant word changes were made.

The SWLS Portuguese version (Marques et al. 2007) was used.

The questionnaire was administered by telephone by trained research assistants. The research assistants were asked to telephone a balanced number of males and females in a 
specific age range. All participants were informed of the anonymity and confidentiality of the survey.

\subsection{Data Analysis}

The first aim of the analyses was intended to investigate the normality of the items of all the measures. All the values for skewness and kurtosis were found under the threshold (skewness $<2$, kurtosis $<7$ ) recommended for running the Confirmatory Factorial Analysis (CFA) with maximum-likelihood estimation. Missing values were, in fact, scarce (under $1 \%$ of the total) and were substituted by the mean value for each item in the measures. Afterwards, reliability, descriptive statistics and inter-correlation matrix were analyzed. We conducted an exploratory factor analysis (EFA) that revealed only one factor explaining $63 \%$ of the variance. However, as the construct had been validated we also performed a CFA. All these preliminary analyses were conducted with SPSS 16. Next, CFA and Multi-group CFA (MCFA) were conducted for the measure of SHS using the five age groups. CFAs and MCFA were performed in order to assess the fit of the unidimensional model for each age group as well as for the overall sample. AMOS 16 was used to run CFA and MCFA. The indices of the model fit considered were: the goodness of fit index (GFI), the comparative fit index (CFI) and the root mean square error of approximation (RMSEA). The GFI can be classified as absolute indices of fit because it basically compares the hypothesized model with no model at all (Hu and Bentler 1995). Values of GFI close to 1.00 are indicative of a good fit. CFI assesses the extent to which the tested model is superior to an alternative model in reproducing the observed covariance matrix (Bentler 1990; McDonald and Marsh 1990). The CFI index varies from 0 to 1 . A value between .92 and .95 is considered well-fitting (Hu and Bentler 1999; Arbuckle 2006; Byrne 2004) and performs well in small samples (Hu and Bentler 1998). The RMSEA introduces a correction for lack of parsimony since, all other things being equal, more complex models are penalized. An RMSEA value of $\leq 0.08$ is a reasonable error of approximation of the population (Arbuckle 2006; Byrne 2004).

\section{Results}

\subsection{Descriptive Statistics, Reliability and Construct Validity of the SHS}

The SHS scale shows a mean of 4.77. This score suggests that the whole sample perceive a moderate sense of subjective happiness. Interestingly, even if a general higher mean value can be registered for the female groups, statistical differences between male and female subjective happiness perception were not found across the groups. Apparently, the youngest and the oldest groups show the highest mean values, respectively $M=4.84$ and $\mathrm{M}=4.85$. However, the post hoc Anova analyses showed that the differences between the age groups are not statistically significant. These results supported previous findings indicating that no significant gender or age differences were observed for the SHS (Lyubomirsky and Lepper 1999; Swami 2008).

Table 1 shows the Cronbach's alpha coefficients and the scale scores of the SHS for each group. The alpha coefficients ranged from .73 (group aged 30-34 years) to .80 (group aged 25-29 years), with a mean of .77 and a value of .77 for the overall sample. Results from previous studies (Lyubomirsky and Lepper 1999) indicated a unidimensional model 
Table 2 Fit indices for the unidimensional factor solution for the SHS

Table 3 Correlation between SHS and SWLS

\begin{tabular}{llll}
\hline Groups & GFI & CFI & RMSEA \\
\hline 1 & .97 & .96 & .17 \\
2 & .98 & .97 & .12 \\
3 & .97 & .98 & .16 \\
4 & .99 & .99 & .10 \\
5 & .98 & .97 & .13 \\
Multi-group & .98 & .97 & .06 \\
\hline
\end{tabular}

\begin{tabular}{lc}
\hline Groups & $\mathrm{r}^{2}$ \\
\hline 1 & .61 \\
2 & .52 \\
3 & .57 \\
4 & .48 \\
5 & .62 \\
Overall & .56 \\
\hline
\end{tabular}

for the SHS scale. Table 2 shows the value of the fit indices considered in this study for CFA and MCFA. Although, the RMSEA values appear to be above the threshold of .08, the other indices show an excellent fit for all the groups, and the RMSEA value for the MCFA indicates a good fit of the model. An explanation for this discrepancy could be given by the recent contribution of Chen et al. (2008). These authors pointed out that RMSEA underestimates the fit of a model for small samples. Afterwards, a multigroup CFA was performed to assess the configural invariance of the unidimensional model across the groups (Byrne 2004). Configural invariance is tested if the basic model structure is invariant across the groups (Byrne 2004). The full configural model had an excellent fit to the data $(\mathrm{GFI}=.98 ; \mathrm{CFI}=.97$; RMSEA $=.06)$. These results reveal that the assumption of configurational invariance was supported. Thus, the unidimensional factor structure of the SHS was supported across the five Portuguese groups.

\subsection{Relationship Between the Construct of Life Satisfaction and Happiness in the Portuguese Culture}

This study also examined the relationship between SHS and SWLS. This provided evidence for the criterion-validity of the SHS measure. Table 3 shows the overall and the groups' correlation coefficients. The correlation between SHS and SWLS in the overall sample is .56 , indicating that the two constructs are generally highly correlated. This result supported previous findings (Lyubomirsky and Lepper 1999; Swami et al. 2009). Interestingly, the correlation seems to vary across the groups. In particular, for the youngest and oldest groups the correlation is stronger, whereas the lowest correlation can be observed for the sample aged 40-44. Finally, the high correlation found between SHS and SWLS provides evidence for the criterion validity of the Portuguese version of the SHS scale. 


\section{Discussion}

The results of this study suggest that the Subjective Happiness Scale can be assessed in Portuguese adults. A large sample from five working Portuguese adult groups was considered to assess the reliability, construct and criterion-related validity of the SHS. Specifically, the SHS showed adequate reliability $(.73<$ Cronbach's alpha $>.80)$ and the MCFA (Multi-group Confirmatory Factorial Analysis) supported the underlying unidimensional theoretical model for the SHS. The criterion-validity of the scale was considered by examining its relationship to SWLS. SHS scores were highly and positively correlated with SWLS scores. These findings supported the criterion validity of the SHS.

The present findings indicate a better fit of the data to the theoretical unidimensional factorial structure for the oldest group. This result would support previous assumptions and findings regarding the fact that it is likely that the construct of happiness, like other social attitude and value constructs, is not completely crystallised until late in life (Gouveia et al. 2009).

In short, this study shows that the SHS Portuguese version exhibits promising values relating to structural and psychometric properties, permitting its continued use in investigation. However, the psychometric properties should be examined further in different populations, such as for example adolescents and adults over 50 years old.

Acknowledgments This research was supported by FCT (Science and Technology Foundation), project PTDC/PSI/73347/2006.

\section{References}

Abdallah, T. (1998). The Satisfaction With Life Scale (SWLS): Psychometric properties in and Arabicspeaking sample. International Journal of Adolescence and Youth, 7, 113-119.

Arbuckle, J. L. (2006). Amos (Version 7.0). Chicago: SPSS, [Computer Program].

Arrindell, W. A., Meeuwesen, L., \& Huyse, F. J. (1991). The Satisfaction With Life Scale: Analysis of factorial invariance across sexes. Personality and Individual Differences, 35, 1255-1260.

Bentler, P. M. (1990). Comparative fit indexes in structural models. Psychological Bulleting, 107, $238-246$.

Blais, M. R., Vallerand, R. J., Pelletier, L. G., \& Briere, N. M. (1990). L'echelle de satisfaction de vie: Validation canadienne-francaise du "Satisfaction With Life Scale". Canadian Journal of Behavioural Science, 21, 210-223.

Bradburn, N. M. (1969). The Structure of Psychological Well-Being. Chicago: Alpine.

Byrne, B. M. (2004). Testing for multi-group invariance using AMOS graphics: A road less travelled. Structural Equation Modeling, 11, 272-300.

Chen, F., Curran, P., Bollen, K., Kirby, J., \& Paxton, P. (2008). An Empirical Evaluation of the Use of Fixed Cutoff Points in the RMSEA Test Statistic in Structural Equation Models. Sociological Methods and Research, 36, 462-494.

Diener, E. (1994). Assessing subjective well-being: Progress and opportunities. Social Indicators Research, $31,103-157$.

Diener, E., \& Diener, M. (1995). Cross cultural correlates of life satisfaction self-esteem. Journal of Personality and Social Psychology, 68, 653-663.

Diener, E., Emmons, R. A., Larsen, R. J., \& Griffin, S. (1985). The Satisfaction with Life Scale. Journal of Personality Assessment, 49, 71-75.

Dupuy, H. J. (1978) The research edition of the general psychological well-being schedule. unpublished manuscript.

Gouveia, V. V., Milfont, T. L., Nunes da Fonseca, P., \& Peçanha de Miranda Coelho, J. A. (2009). Life Satisfaction in Brazil: Testing the Psychometric Properties of the Satisfaction With Life Scale (SWLS) in Five Brazilian Samples. Social Indicators Research, 90, 267-277.

Hu, L. T., \& Bentler, P. (1995). Evaluating model fit. In R. H. Hoyle (Ed.), Structural Equation Modeling. Concepts, Issues, and Applications (pp. 76-99). London: Sage. 
Hu, L., \& Bentler, P. M. (1998). Fit indexes in covariance structure modeling: sensitivity to under parameterized model misspecification. Psychological Methods, 3, 424-453.

Hu, L., \& Bentler, P. M. (1999). Cutoff criterion for fit indexes in covariance structure analysis: Conventional criteria versus new alternatives. Structural Equation Modeling, 6, 1-55.

Lyubomirsky, S., \& Lepper, H. S. (1999). A measure of subjective happiness: preliminary reliability and construct validation. Social Indicators Research, 46, 137-155.

Marques, S. C., Pais-Ribeiro, J. L., \& Lopez, S. J. (2007). Validation of a Portuguese Version of the Students' Life Satisfaction Scale. Applied Research in Quality of Life, 2, 83-94.

McDonald, R. P., \& Marsh, H. W. (1990). Choosing a multivariate model: noncentrality and goodness of fit. Psychological Bulletin, 107, 247-255.

Neto, F. (1993). The Satisfaction With Life Scale: Psychometrics properties in an adolescent sample. Journal of Youth and Adolescence, 22, 125-134.

Ryan, R. M., Little, T. D., Sheldon, K. M., Timoshina, E., \& Deci, E. L. (1999). The American dream in Russia: extrinsic aspiration and well-being in two cultures. Personality and Social Psychology Bulletin, $25,1509-1524$.

Shimai, S., Otake, K., Utsuki, N., Ikemi, A., \& Lyubomirsky, S. (2004). Development of a Japanese version of the Subjective Happiness Scale (SHS), and examination of its validity and reliability. Nippon Koshu Eisei Zasshi, 51, 845-853.

Swami, V. (2008). Translation and validation of the Malay Subjective Happiness Scale. Social Indicators Research, 88, 347-353.

Swami, V., \& Chamorro-Premuzic, T. (2009). Psychometric Evaluation of the Malay Satisfaction With Life Scale. Social Indicators Research, 92, 25-33.

Swami, V., Stieger, S., Voracek, M., Dressler, S. G., Eisma, L., \& Furnham, A. (2009). Psychometric evaluation of the Tagalog and German Subjective Happiness Scale and a cross-cultural comparison. Social Indicators Research, 93, 393-406. 